\title{
Lymphangitis Carcinomatosa: Report of a Case and Review of Literature
}

\author{
Anand Raja • Ramakrishnan Ayloor Seshadri • \\ Shirley Sundersingh
}

Received: 9 February 2010 / Accepted: 30 January 2011 /Published online: 5 March 2011

(C) Indian Association of Surgical Oncology 2011

\section{Introduction}

Lymphangitis carcinomatosa describes a condition of diffuse infiltration of the lymphatics of both the lungs by malignant cells. It may be the only manifestation of an occult malignancy and should be included in the differential diagnosis of dyspnoea, especially in young patients in whom this diagnosis is usually not suspected. The diagnosis rests on a high index of suspicion in the setting of progressive respiratory failure. In this article we review the clinical presentation and the diagnostic methods. We also review the relevant literature pertaining to this rare entity.

\section{The Case}

A 31 year old lady presented with symptoms of pain in the abdomen and vomiting of 1 month duration. She also had nonproductive cough for 2 weeks. Clinical examination revealed her to be in ECOG performance status 2, with an ill defined lump palpable in the epigastrium. There was no

Key messages: Lymphangitis carcinomatosa is a rare entity and may be the presenting feature of an underlying malignancy. It requires a high index of suspicion for diagnosis.

A. Raja $\cdot$ R. A. Seshadri $(\bowtie)$

Department of Surgical Oncology, Cancer Institute (WIA),

Annexe Campus, No. 18, Sardar Patel Road, Guindy,

Chennai 600036, India

e-mail: ram_a_s@yahoo.com

S. Sundersingh

Department of Pathology, Cancer Institute(WIA),

Annexe Campus, No. 18, Sardar Patel Road, Guindy,

Chennai 600036, India ascites, jaundice, palpable cervical nodes or pelvic deposits. Respiratory system examination was normal. A computerized tomography (CT) scan of the abdomen and pelvis revealed thickening of the distal stomach with perigastric nodes. She was scheduled for an endoscopic evaluation, but she presented to the emergency on the second day with acute breathlessness. Chest radiographs revealed diffuse haziness in both lower lung fields but there was no evidence of pleural effusion or pulmonary metastatic nodules. A presumptive diagnosis of pneumonia was made and she was started on broad spectrum antibiotics. She had low oxygen saturation and needed nasal oxygen supplementation. Arterial blood gas analysis was suggestive of Type I respiratory failure. Her general condition did not permit evaluation by CT chest or endoscopy. Sputum culture showed growth of Klebsiella Pneumoniae for which appropriate antibiotic cover was initiated. The sputum examination did not reveal acid fast bacilli. Despite these measures, her respiratory distress progressively worsened. Subsequent chest radiographs done over the next 3 days showed progressive involvement of the middle and upper zones of both lung fields by interstitial infiltrates (Fig. 1). As she was not responding to antibacterial therapy, empirical antifungal and anti viral drugs were added. She had to be intubated and supported with mechanical ventilation due to progressive respiratory failure. Her condition however continued to deteriorate and she died of respiratory failure 4 days after admission. Post mortem biopsies from both the lungs showed lung parenchyma with the alveoli lined and filled with diffuse sheaths of atypical round to oval cells with pale cytoplasm and irregular hyperchromatic nuclei with a mitosis of $1-3 / \mathrm{hpf}$. Peri-epithelial and lymphatic tumor emboli were also seen. All these features suggested pulmonary lymphangitis carcinomatosa of gastric origin (Fig. 2). 
Fig. 1 Serial Chest $\mathrm{X}$ rays day 1 through day 4 showing progressive of the lung fields
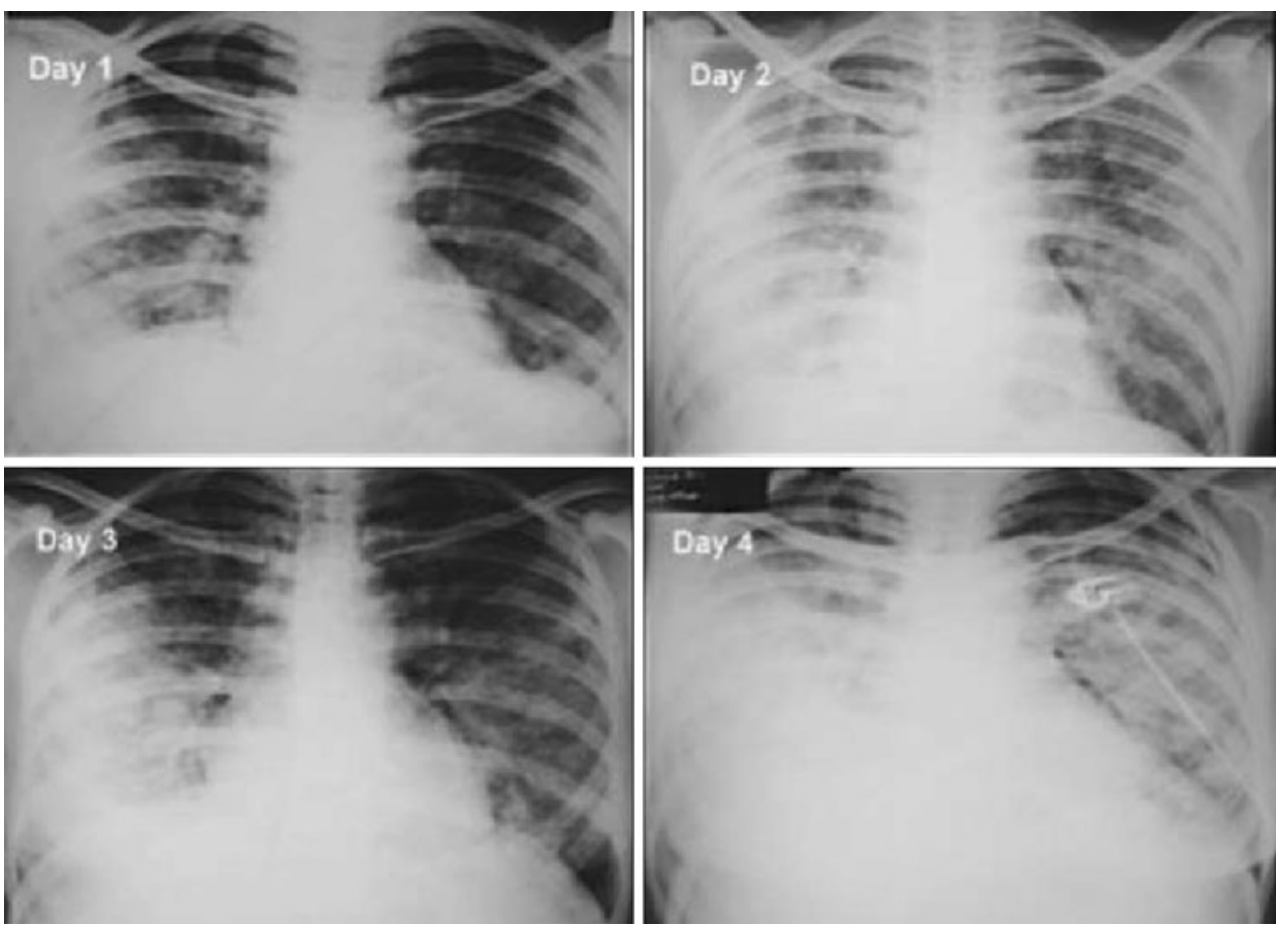

\section{Discussion}

Lymphangitis carcinomatosa is a term introduced by Troisier in 1873 to describe diffuse infiltration of the lymphatics of both the lungs by malignant cells. The condition affects males more than the females (60:40), and affects a younger population than that affected with most malignancies [1].

Intra-thoracic metastasis occurs in 30 to $40 \%$ of patients with malignant disease. In this group 6-8\% develop lymphangitis carcinomatosa [1].

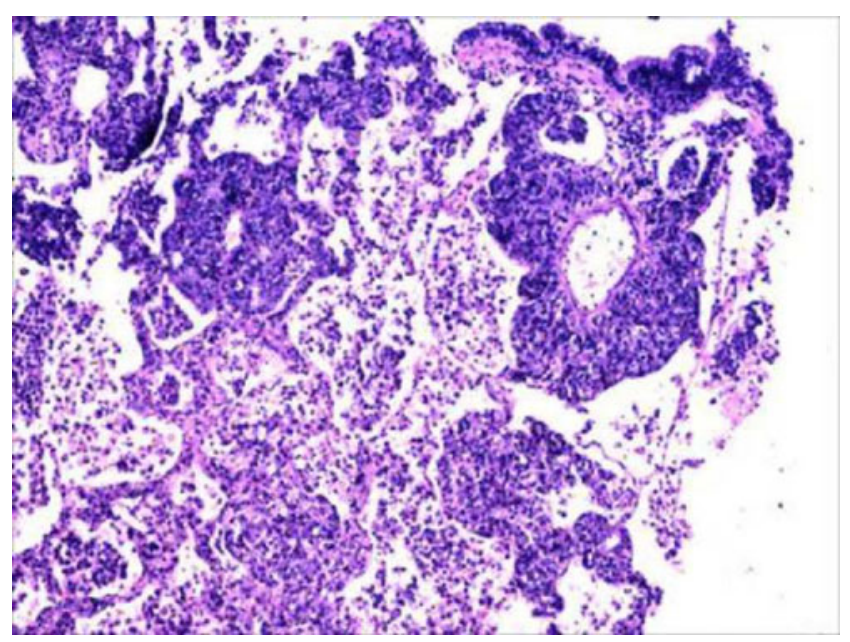

Fig. 2 Lung parenchyma with dilated lymphatic channels filled with malignant appearing cells suggestive of metastatic adenocarcinoma
There are two theories as to how this condition occurs. The first states that there is haematogenous metastasis producing obliterative endarteritis and then tumor cells subsequently egress through vascular walls into the perivascular lymphatics. The other theory states that there may be diffuse retrograde permeation and embolization of lymphatics after involvement of the hilar lymph nodes [2]. Lymphangitis carcinomatosa may precede or obscure or dominate any local symptoms and present as a diagnostic problem in dyspnoea. It may also occur during the evolution of a growth, which has already been identified during life, in which case diagnosis becomes relatively easier [1]. The clinical presentation and progression of our patient agrees with that reported by other authors who noted incapacitating unremitting dyspnoea as the most common and distressing feature of the patient [3]. On chest radiography abnormalities are found to be slightly more common in the right lung than in the left. No single radiographic change is diagnostic, but the combination of abnormalities is sufficiently characteristic to suggest the diagnosis in about half the cases. Nodular shadows are most commonly seen but diffuse shadows, hilar lymphadenopathy and pleural effusion are additional findings [4]. The high resolution CT scan with $1.5 \mathrm{~mm}$ cuts is more useful than conventional CT. The distinctive changes are thickening and irregularity of the linear patterns and changes in a broncho-alveolar and peri-lobular pattern [1]. The average arterial pO2 is $42-46 \mathrm{mmHg}$ [5]. The biopsy can be proved 
by trans bronchial biopsies or needle biopsy or by autopsy studies [2]. There are currently no effective strategies to treat lymphangitis carcinomatosa. However steroid administration may produce symptomatic improvement mainly by alleviating breathlessness [1]. The prognosis remains poor, with most patients dying within 3 to 12 months [1].

Lymphangitis carcinomatosa is a rare clinico-pathological entity. It should be included in the differential diagnosis of patients with acute progressive respiratory failure and malignancy, not responding to conventional medication. A high index of suspicion is required to diagnose this condition.

\section{References}

1. Bruce DM, Heys SD, Eremin O (1996) Lymphangitis carcinomatosa: a literature review. J R Coll Surg Edinb 41:7-13

2. Chandler GN, Telling M (1952) Lymphangitis carcinomatosa. $\mathrm{Br}$ Med J 2:639-641

3. Mendeloff AI (1951) Severe asthamatic dyspnoea as the sole presenting symptom of generalized endolymphatic carcinomatosis. Ann Intern Med 34:881-888

4. David HT (1964) Radiological appearances of lymphangitis carcinomatsa of the lung. Thorax 19:251-260

5. Dennstedt FE, Greenberg SD, Kim HS, Weilbaecher DG, Bloom K (1983) Pulmonary lymphangitic carcinomatosis from occult stomach carcinoma in young adults: an unusual cause of dyspnea. Chest $84: 787-788$ 